\title{
The ATLAS Conditions Database Model for the Muon Spectrometer
}

\author{
Monica Verducci ${ }^{1}$ \\ INFN Sezione di Roma \\ P.le Aldo Moro 5,00185 Rome, Italy \\ E-mail: monica.verducci@cern.ch

\section{on behalf of the ATLAS Muon Collaboration}

\begin{abstract}
The Muon System has extensively started to use the LCG conditions database project 'COOL' as the basis for all its conditions data storage both at CERN and throughout the worldwide collaboration as decided by the ATLAS Collaboration. The management of the Muon COOL conditions database will be one of the most challenging applications for Muon System, both in terms of data volumes and rates, but also in terms of the variety of data stored. The Muon conditions database is responsible for almost all of the 'non-event' data and detector quality flags storage needed for debugging of the detector operations and for performing reconstruction and analysis. The COOL database allows database applications to be written independently of the underlying database technology and ensures long-term compatibility with the entire ATLAS Software. COOL implements an interval of validity database, i.e. objects stored or referenced in COOL have an associated start and end time between which they are valid, the data is stored in folders, which are themselves arranged in a hierarchical structure of folder sets. The structure is simple and mainly optimized to store and retrieve object(s) associated with a particular time. In this work, an overview of the entire Muon conditions database architecture is given, including the different sources of the data and the storage model used. In addiction the software interfaces used to access to the conditions data are described, more emphasis is given to the Offline Reconstruction framework ATHENA and the services developed to provide the conditions data to the reconstruction.
\end{abstract}

XII Advanced Computing and Analysis Techniques in Physics Research

Erice, Italy

3-7 November, 2008

\footnotetext{
${ }^{1}$ Speaker
} 


\section{Introduction}

The ATLAS Muon Spectrometer is the outer part of the ATLAS detector and is designed to detect charged particles exiting the barrel and end-cap calorimeters and to measure their momentum in the pseudorapidity range $|\eta|<2.7$. It is also designed to trigger on these particles in the region $|\eta|<2.4$ [1].

The quality of the muon measurement has been one of the guiding design criteria for the ATLAS experiment, the performance goal is a stand-alone transverse momentum resolution of approximately $10 \%$ for $1 \mathrm{TeV}$ tracks, which translates into a sagitta along the $\mathbf{z}$ (beam) axis of about $500 \mu \mathrm{m}$, to be measured with a resolution of $\sim 60 \mu \mathrm{m}$.

This concern is reflected by the choice of the main components of the muon spectrometer: a system of three large superconducting air-core toroid magnets, precision tracking detectors with $\sim 60 \mu \mathrm{m}$ intrinsic resolution, and a powerful dedicated trigger system.

Muon chamber planes are attached to the toroids to measure the muon trajectories. In the barrel, the natural layout consists of three layers of chambers: at the inner and outer edges of the magnetic volume and in the mid-plane to measure the sagitta. In the forward direction the chambers are placed at the front and back faces of the toroid cryostats, with a third layer against the cavern wall, to maximize the lever-arm of the point-angle measurement. The high-precision chambers are complemented with an independent fast trigger chamber system.

The precision momentum measurement is performed by the Monitored Drift Tube chambers (MDT's) and the Cathode-Strip Chambers (CSC's).

The MDT chambers cover the pseudorapidity range $|\eta|<2.7$ (except in the innermost end-cap layer where their coverage is limited to $|\eta|<2.0$ ). These chambers consist of three to eight layers of drift tubes, operated at an absolute pressure of $3 \mathrm{bar}$, which achieve an average resolution of $80 \mu \mathrm{m}$ per tube, or about $35 \mu \mathrm{m}$ per chamber.

In the forward region $(2<|\eta|<2.7)$, CSC chambers are used in the innermost tracking layer due to their higher rate capability and time resolution.

The precision-tracking chambers have therefore been complemented by a system of fast trigger chambers capable of delivering track information within a few tens of nanoseconds after the passage of the particle. In the barrel region $(|\eta|<1.05)$, Resistive Plate Chambers (RPC) were selected for this purpose, while in the end-cap $(1.05<|\eta|<2.4)$ Thin Gap Chambers (TGC) were chosen.

The conditions data will be used during the ATLAS data taking, reconstruction and subsequent processing to describe the environments in which the events have been taken. These data have many different origins, and are stored in many different types of databases: configurations database and a conditions database see [2].

The configuration database will store all the data needed at the start of the run, including sub-detector hardware and software configuration. The conditions database will store all the parameters describing run conditions and logging, all the data which will be accessed offline, i.e. by the reconstruction or analysis software. 
The conditions database is closely related to the configuration database, needed to set up and run the detector hardware and associated online and event selection software.

Conditions data varies with time, and is usually characterized by an "interval of validity” (IoV). It includes data archived from the ATLAS detector control system (DCS), online book-keeping data, online and offline calibration and alignment data, and monitoring data characterizing the performance of the detector and software during any particular period of time.

In the figure 1 there is a draft of the connections between the configuration and the conditions database in the ATLAS offline and online environment.

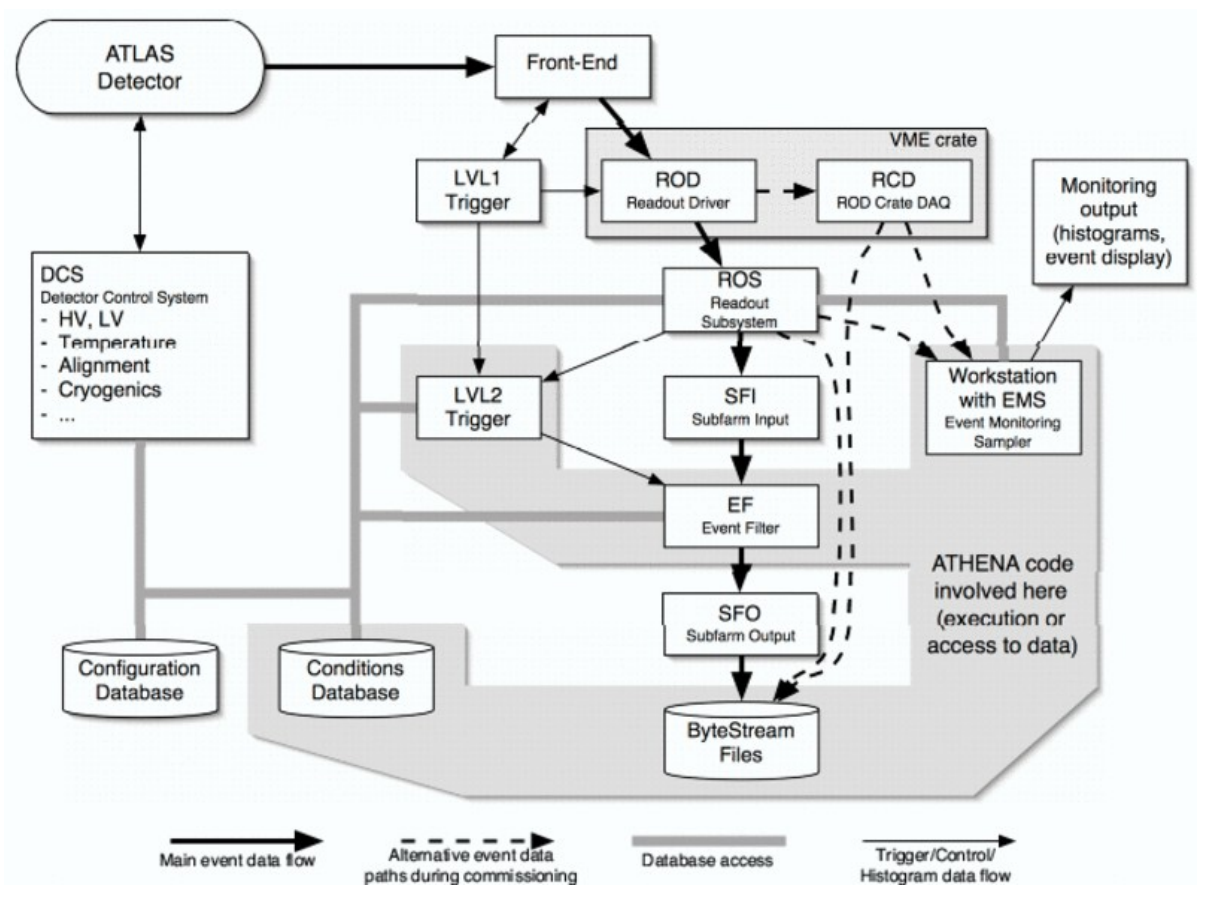

Fig.1: Data Flow of the Events and Conditions Data in the ATLAS experiment. The configurations and the conditions databases are shown (on the left of the picture) and their links with the Detector Hardware and Software of the experiment.

\section{The ATLAS Muon Conditions Data}

The Muon conditions data have different sources both from the online side and from the offline data quality analysis including the calibration and alignment parameters.

The online data primary define the configuration setup of the data taking (DAQ parameters) and of the Detector Control System (DCS), including hardware monitor values as: temperatures, gas pressure, voltage, etc. 
Calibration and alignment constants come from analysis algorithms running on a dedicated muon calibration stream coming from the second level of the trigger $[3,4]$.

Finally, the data quality information, summarizing the quality flags from the online and offline sides in a common result to be given to the reconstruction, for example detector flag as 'dead status' is a merge of this status for the online, offline and calibration streams.

Each subdetector has its own database architecture and its own schema inside the ATLAS conditions servers: ATONR and ATLR, Oracle RAC running in the CERN computer centre (offline and online servers) and at Tier-1s. The folders structures, as the amount of the data, are different due to the different hardware layouts and analysis approaches of the detectors.

The amount of the data for each subdetector depends on the different role and strategy in the tracking algorithm, the data varies strictly with the time.

The Muon conditions deployment is in line with the entire ATLAS project.

\section{The ATLAS conditions database}

The ATLAS conditions database is based on Oracle DB, all the conditions database, in particular for the offline reconstruction, is implemented using COOL technology [5]. COOL, an LCG product is a library to manage conditions data in terms of Interval of Validity (IoV), versions and tags, using CORAL as backend. CORAL allows database applications to be written independently of the underlying database technology (this means that COOL databases can be stored in Oracle, SQLite or MySQL), see for more details [5].

Moreover, the COOL API has been integrated into the ATLAS online software. Several special-purpose higher level interfaces are also being developed, including the Condition Database Interface (CDI) for archiving Information System (IS) data to COOL, the PVSS to COOL interface for archiving Detector Control System (DCS) data, and specialized interfaces for saving monitoring data.

The objects stored or referenced in COOL have an associated start and end time between which they are valid (IoV).

COOL data is stored in folders, which are themselves arranged in a hierarchical structure of folder sets. Within each folder, several objects of the same type are stored, each with its interval of validity range. These times are specified either as run/event, or as absolute timestamps, and the choice between formats is made according to meta-data associated with each folder. The objects in COOL folders can be optionally identified by a channel number (or channel ID) within the folder. Each channel has its own intervals of validity, but all channels can be dealt with together in bulk updates or retrievals.

COOL implements each folder as a relational database table, with each stored object corresponding to a row in the table. COOL creates columns for the start and end times of each object, and optionally the channel ID and tag if used. Several other columns are also created (e.g. insertion time and object ID), to be used internally by the COOL system, but these are generally of no concern to the user.

The payload columns (where the data are stored) are defined by the user when the table is created. In ATLAS, the payload data can be stored in the three following ways. 
The payload data can be stored directly in one or more payload columns (inline data), where the columns directly represent the data being stored (e.g. a mixture of float and integer values in the columns representing status and parameter information).

In second way, the payload data (in this case a single column) can be used to reference data stored elsewhere. This reference can be a foreign key to another database table, or a reference to something outside of COOL - e.g. a POOL object reference allowing an external object to be associated to intervals of validity.

A third approach involves storing the data as an inline CLOB in the database, i.e. defining the payload to be a large character object (CLOB) which has an internal structure invisible to the COOL database. COOL is then responsible only for storing and retrieving the CLOB, and its interpretation is up to the client code.

The retrieving and storing of the data inside a reconstruction job in the Athena framework (offline reconstruction framework) is possible using the IOVService, a software interface between the COOL DB and the reconstruction algorithms via IOV range.

\section{Conclusions}

In this paper, an overview of the Muon conditions database has been given.

The Model has been tested during the Computing and Detector Commissioning.

During the Cosmics tests a full chain test has been performed, including the production of the calibration and alignment constant subsequent storage in the COOL folders and later access during a reconstruction job.

The results have been promising, and the Muon conditions schema is now in production, additional features will be implemented in the next months.

\section{References}

[1] ATLAS Muon Collaboration, "The ATLAS Muon Spectrometer Technical Design Report”, CERNLHCC/97-22 [Online], May 31 1997,

[2] ATLAS Collaboration, ATLAS Computing Technical Design Report, CERN/LHCC/2005-022, (2005)

[3] E.Pasqualucci, et al. "Muon detector calibration in the ATLAS experiment: data extraction and distribution”, in Proceedings of Computingin High Energy and Nuclear Physics CHEP06, Mumbai,India, 13-17 Feb. 2006

[4] M. Verducci, “ATLAS Conditions Database and Calibration Streams”, Nuclear Physics B (Proc.Suppl.) 172 (2007) 250-252

[5] A. Valassi, COOL web page, Available at http://lcgapp.cern.ch/project/CondDB/ 